\title{
Comprehensive Analysis of Profitability of Modern Logistics Enterprise
}

\author{
An Example of Transportation, Warehousing, Postal Service Listed Company
}

\author{
Yinghong Zhao \\ Shenyang Normal University \\ Shenyang,China
}

\begin{abstract}
Modern logistics enterprise is a collection of transportation, storage, handling, packaging, circulation processing, and distribution. Factor analysis method and the financial data of modern logistics company, such as transportation, warehousing and postal service, has been used to comprehensively analyse the profitability of 73 listed companies. The results show that the comprehensive profitability of transportation industry is stronger than others, and the stronger transportation enterprises are mainly located in coastal and economically developed areas, such as Guangdong, Hainan, Fujian, Shandong, Jiangsu, Shanghai, and so on.
\end{abstract}

Keywords-modern logistics enterprises; profitability; comprehensive analysis ; KMO test; Bartlett sphericity test

\section{INTRODUCTION}

Modern Logistics Enterprises is based on traditional logistics enterprises, brings in high technology, such as information, to meet the logistics needs of customers, and has functions of transportation, storage, handling, packaging, circulation processing, distribution, etc. Logistics distribution mode of modern logistics enterprises has characteristics of informatization, automation, modernization, socialization, intelligent, rationalization and simplistic.This can reduce inventory of production enterprises, accelerate the capital turnover, improve the efficiency of logistics, reduce logistics cost, and stimulate the social demand.

\author{
Danmeng Gao \\ Shenyang University of Chemical Technology, \\ Shenyang,China
}

\section{FinANCIAL EVALUATION INDEX OF PROFITABILITY} OF MODERN LOGISTICS ENTERPRISE

\section{A. Index Selection}

Corporate profitability can be reflected from multiple perspectives and multiple profiles. The selection of index should follow the principle as comprehensive, scientific, comprehensive, systematic, operability, and dynamic and static combination to comprehensively and correctly reflect the profitability of enterprises.In this study, 10 indexes have been selected, including Netprfrt、ROA、 ROE 、 ROAgrrt 、 Netassgrrt 、 Netprfgrrt 、 Currat 、 Totassrat、Currt and Qckrt.

\section{B. Sample Index Data Source}

Since the 2013 annual report data was reported in late April or early May in 2014, this study selected all listed companies of modern logistics enterprises as sample, acquired data from RESSET for three consecutive years from 2010 to 2012. ST and listed company that the data is incomplete was rejected. The data is analysed by SPSS 19.0 .

\section{FACTOR ANALYSIS OF PROFITABILITY OF MODERN LOGISTICS ENTERPRISE NONDIMENSIONALIZED THE ORIGINAL DATA FIRST.}

\section{A KMO and Bartlett sphericity test.}

KMO value was used to verify the applicability of factor analysis (see Table 1). 
TABLE I. KMO AND BARTLETT'S TEST

\begin{tabular}{|c|c|c|c|c|}
\hline & & $\begin{array}{l}20 \\
10\end{array}$ & $\begin{array}{l}20 \\
11\end{array}$ & $\begin{array}{l}20 \\
12\end{array}$ \\
\hline Kaiser-Meyer-Olkin & & 0. & 0 . & 0 . \\
\hline Measure of Sampling & & 75 & 73 & 78 \\
\hline Adequacy. & & 3 & 9 & 9 \\
\hline Bartlett's Test of Sphericity & $\begin{array}{l}\text { Approx. } \\
\text { Chi-Square }\end{array}$ & $\begin{array}{r}75 \\
0 . \\
5\end{array}$ & $\begin{array}{r}72 \\
8\end{array}$ & $\begin{array}{r}82 \\
6 . \\
5\end{array}$ \\
\hline & df & 46 & 46 & 46 \\
\hline & Sig. & $\begin{array}{r}0 . \\
00\end{array}$ & $\begin{array}{c}0 . \\
00\end{array}$ & $\begin{array}{l}0 . \\
00\end{array}$ \\
\hline
\end{tabular}

KMO values were respectively $0.753,0.739,0.789$ from 2010 to 2012 , in line with the requirements of factor analysis and concomitant probability of bartlett sphericity test was $0.00, \mathrm{p}<0.05$, which showed that indexes used in the study had strong correlation, and the factor analysis was effective.

\section{B The Determination of Common Factor}

The result offactor analysis of were presented in Table 2, Table 3, Table 4.

TABLE II. TOTAL VARIANCE EXPLAINTED OF FACTOR ANALYSIS IN 2010

\begin{tabular}{|c|c|c|c|c|c|c|c|c|c|}
\hline & \multicolumn{3}{|c|}{$\begin{array}{c}\text { Initial } \\
\text { Eigenvalues }\end{array}$} & \multicolumn{3}{|c|}{$\begin{array}{l}\text { Sums of } \\
\text { Squared } \\
\text { Loadings }\end{array}$} & \multicolumn{3}{|c|}{$\begin{array}{c}\text { Rotation Sums } \\
\text { of Squared } \\
\text { Loadings }\end{array}$} \\
\hline $\begin{array}{c}\text { Co } \\
\text { mp } \\
\text { one } \\
\text { nt }\end{array}$ & $\begin{array}{l}\text { ot } \\
\text { al }\end{array}$ & $\begin{array}{l}\text { of } \\
\mathrm{V} \\
\text { ari } \\
\text { an } \\
\text { ce }\end{array}$ & $\begin{array}{l}\text { Cum } \\
\text { ulati } \\
\text { ve\% }\end{array}$ & $\begin{array}{l}\mathrm{T} \\
\mathrm{o} \\
\mathrm{t} \\
\mathrm{a} \\
1\end{array}$ & $\begin{array}{l}\% \\
\text { of } \\
\mathrm{V} \\
\text { ari } \\
\text { an } \\
\text { ce }\end{array}$ & $\begin{array}{l}\text { Cum } \\
\text { ulati } \\
\text { ve } \%\end{array}$ & $\begin{array}{l}0 \\
\mathrm{t} \\
\mathrm{a} \\
1\end{array}$ & $\begin{array}{l}\% \\
\text { of } \\
\mathrm{V} \\
\text { ari } \\
\text { an } \\
\text { ce }\end{array}$ & $\begin{array}{l}\text { Cum } \\
\text { ulati } \\
\text { ve } \%\end{array}$ \\
\hline
\end{tabular}

\begin{tabular}{|c|c|c|c|c|c|c|c|c|c|}
\hline 1 & 8 & $\begin{array}{c}28 \\
.3 \\
60\end{array}$ & $\begin{array}{c}28.3 \\
60\end{array}$ & $\begin{array}{l}. \\
8 \\
3\end{array}$ & $\begin{array}{l}28 \\
.3 \\
60\end{array}$ & $\begin{array}{c}28.3 \\
60\end{array}$ & $\begin{array}{l}. \\
3 \\
9 \\
6\end{array}$ & $\begin{array}{c}25 \\
.9 \\
60\end{array}$ & $\begin{array}{c}25.9 \\
60\end{array}$ \\
\hline 2 & $\begin{array}{l}2 . \\
4 \\
6\end{array}$ & $\begin{array}{c}24 \\
.6 \\
93\end{array}$ & $\begin{array}{c}53.0 \\
53\end{array}$ & $\begin{array}{l}. \\
4 \\
6\end{array}$ & $\begin{array}{l}24 \\
.6 \\
93\end{array}$ & $\begin{array}{c}53.0 \\
53\end{array}$ & $\begin{array}{l}2 \\
. \\
0 \\
9 \\
2\end{array}$ & $\begin{array}{c}20 \\
.9 \\
17\end{array}$ & $\begin{array}{c}46.8 \\
76\end{array}$ \\
\hline 3 & $\begin{array}{l}1 . \\
3\end{array}$ & $\begin{array}{l}15 \\
.9 \\
60\end{array}$ & $\begin{array}{c}69.0 \\
12\end{array}$ & $\begin{array}{l}. \\
3 \\
9\end{array}$ & $\begin{array}{l}15 \\
.9 \\
60\end{array}$ & $\begin{array}{c}69.0 \\
12\end{array}$ & $\begin{array}{l}1 \\
. \\
8 \\
6 \\
6\end{array}$ & $\begin{array}{c}18 \\
.6 \\
57\end{array}$ & $\begin{array}{c}65.5 \\
33\end{array}$ \\
\hline 4 & $\begin{array}{l}1 . \\
1\end{array}$ & $\begin{array}{l}11 \\
.6 \\
03\end{array}$ & $\begin{array}{c}80.6 \\
16\end{array}$ & $\begin{array}{l}. \\
1 \\
6\end{array}$ & $\begin{array}{l}11 \\
.6 \\
03\end{array}$ & $\begin{array}{c}80.6 \\
16\end{array}$ & $\begin{array}{l}1 \\
. \\
5 \\
0 \\
8\end{array}$ & $\begin{array}{l}15 \\
.0 \\
83\end{array}$ & $\begin{array}{c}80.6 \\
16\end{array}$ \\
\hline 5 & $\begin{array}{l}.8 \\
0 \\
1\end{array}$ & $\begin{array}{c}8 . \\
00 \\
5\end{array}$ & $\begin{array}{c}88.6 \\
21\end{array}$ & & & & & & \\
\hline 6 & $\begin{array}{l}.7 \\
5 \\
1\end{array}$ & $\begin{array}{c}5 . \\
50 \\
9\end{array}$ & $\begin{array}{c}94.1 \\
30\end{array}$ & & & & & & \\
\hline 7 & $\begin{array}{l}.3 \\
2 \\
5\end{array}$ & $\begin{array}{c}3 . \\
24 \\
7\end{array}$ & $\begin{array}{c}97.3 \\
77\end{array}$ & & & & & & \\
\hline 8 & $\begin{array}{l}.2 \\
2\end{array}$ & $\begin{array}{l}2 . \\
21 \\
5\end{array}$ & $\begin{array}{c}99.5 \\
93\end{array}$ & & & & & & \\
\hline 9 & .0 & $\begin{array}{l}.2 \\
97\end{array}$ & $\begin{array}{c}99.8 \\
90\end{array}$ & & & & & & \\
\hline 10 & .0 & $\begin{array}{l}.1 \\
10\end{array}$ & $\begin{array}{r}100 . \\
000\end{array}$ & & & & & & \\
\hline
\end{tabular}


TABLE III. TOTAL VARIANCE EXPLAINTED OF FACTOR ANALYSIS IN 2011

\begin{tabular}{|c|c|c|c|c|c|c|c|c|c|}
\hline & \multicolumn{3}{|c|}{$\begin{array}{c}\text { Initial } \\
\text { Eigenvalues }\end{array}$} & \multicolumn{3}{|c|}{$\begin{array}{l}\quad \text { Sums of } \\
\text { Squared } \\
\text { Loadings }\end{array}$} & \multicolumn{3}{|c|}{$\begin{array}{c}\text { Rotation Sums } \\
\text { of Squared } \\
\text { Loadings }\end{array}$} \\
\hline $\begin{array}{l}\text { Co } \\
\text { mp } \\
\text { one } \\
\text { nt }\end{array}$ & $\begin{array}{l}\mathrm{o} \\
\mathrm{t} \\
\mathrm{a} \\
\mathrm{l}\end{array}$ & $\begin{array}{l}\% \\
\text { of } \\
\mathrm{V} \\
\text { ari } \\
\text { an } \\
\text { ce }\end{array}$ & $\begin{array}{l}\text { Cum } \\
\text { ulati } \\
\text { ve } \%\end{array}$ & $\begin{array}{l}\mathrm{T} \\
\mathrm{o} \\
\mathrm{t} \\
\mathrm{a} \\
1\end{array}$ & $\begin{array}{l}\% \\
\text { of } \\
\mathrm{V} \\
\text { ari } \\
\text { an } \\
\text { ce }\end{array}$ & $\begin{array}{l}\text { Cum } \\
\text { ulati } \\
\text { ve } \%\end{array}$ & $\begin{array}{l}\mathrm{T} \\
\mathrm{o} \\
\mathrm{t} \\
\mathrm{a} \\
1\end{array}$ & $\begin{array}{c}\% \\
\text { of } \\
\text { Va } \\
\text { ria } \\
\mathrm{nc} \\
\mathrm{e}\end{array}$ & $\begin{array}{l}\text { Cum } \\
\text { ulati } \\
\text { ve } \%\end{array}$ \\
\hline 1 & 6 & $\begin{array}{l}26 \\
.1 \\
28\end{array}$ & $\begin{array}{c}26.1 \\
28\end{array}$ & 6 & $\begin{array}{l}26 \\
.1 \\
28\end{array}$ & $\begin{array}{c}26.1 \\
28\end{array}$ & 3 & $\begin{array}{l}23 \\
.2 \\
60\end{array}$ & $\begin{array}{c}23.2 \\
60\end{array}$ \\
\hline 2 & 0 & $\begin{array}{l}24 \\
.0 \\
71\end{array}$ & $\begin{array}{c}50.1 \\
98\end{array}$ & 4 & $\begin{array}{l}24 \\
.0 \\
71\end{array}$ & $\begin{array}{c}50.1 \\
98\end{array}$ & 1 & $\begin{array}{r}21 \\
.8 \\
31\end{array}$ & $\begin{array}{c}45.0 \\
91\end{array}$ \\
\hline 3 & 4 & $\begin{array}{l}19 \\
.4 \\
39\end{array}$ & $\begin{array}{c}69.6 \\
37\end{array}$ & $\begin{array}{l}. \\
9 \\
4\end{array}$ & $\begin{array}{l}19 \\
.4 \\
39\end{array}$ & $\begin{array}{c}69.6 \\
37\end{array}$ & $\begin{array}{l}0 \\
5\end{array}$ & $\begin{array}{r}20 \\
.5 \\
13\end{array}$ & $\begin{array}{c}65.6 \\
04\end{array}$ \\
\hline 4 & 2 & $\begin{array}{l}12 \\
.2 \\
18\end{array}$ & $\begin{array}{c}81.8 \\
55\end{array}$ & 2 & $\begin{array}{l}12 \\
.2 \\
18\end{array}$ & $\begin{array}{c}81.8 \\
55\end{array}$ & . & $\begin{array}{l}16 \\
.2 \\
51\end{array}$ & $\begin{array}{c}81.8 \\
55\end{array}$ \\
\hline 5 & 0 & $\begin{array}{c}8 . \\
04 \\
2\end{array}$ & $\begin{array}{c}89.8 \\
98\end{array}$ & & & & & & \\
\hline 6 & 0 & $\begin{array}{l}5 . \\
08 \\
0\end{array}$ & $\begin{array}{c}94.9 \\
77\end{array}$ & & & & & & \\
\hline 7 & $\begin{array}{l}7 \\
9\end{array}$ & $\begin{array}{l}2 . \\
78 \\
7\end{array}$ & $\begin{array}{r}97.7 \\
64\end{array}$ & & & & & & \\
\hline
\end{tabular}

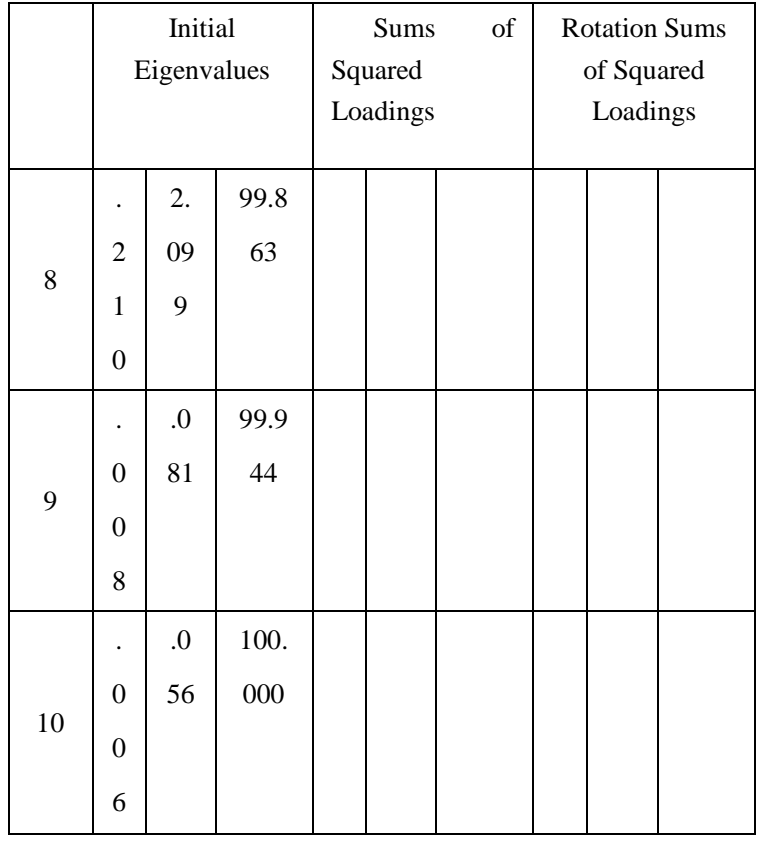

TABLE IV. TOTAL VARIANCE EXPLAINTED OF FACTOR ANALYSIS IN 2012

\begin{tabular}{|c|c|c|c|c|c|c|c|c|c|}
\hline & \multicolumn{3}{|c|}{$\begin{array}{c}\text { Initial } \\
\text { Eigenvalues }\end{array}$} & \multicolumn{3}{|c|}{$\begin{array}{l}\text { Sums of } \\
\text { Squared } \\
\text { Loadings }\end{array}$} & \multicolumn{3}{|c|}{$\begin{array}{c}\text { Rotation Sums } \\
\text { of Squared } \\
\text { Loadings }\end{array}$} \\
\hline $\begin{array}{l}\text { Co } \\
\text { mp } \\
\text { one } \\
\text { nt }\end{array}$ & $\begin{array}{l}\mathrm{T} \\
\mathrm{o} \\
\mathrm{t} \\
\mathrm{a} \\
\mathrm{l}\end{array}$ & $\begin{array}{l}\% \\
\text { of } \\
\mathrm{V} \\
\text { ari } \\
\text { an } \\
\text { ce }\end{array}$ & $\begin{array}{l}\text { Cum } \\
\text { ulati } \\
\text { ve } \%\end{array}$ & $\begin{array}{l}0 \\
\mathrm{t} \\
\mathrm{a} \\
1\end{array}$ & $\begin{array}{l}\% \\
\text { of } \\
\mathrm{V} \\
\text { ari } \\
\text { an } \\
\text { ce }\end{array}$ & $\begin{array}{l}\text { Cum } \\
\text { ulati } \\
\text { ve } \%\end{array}$ & $\mathrm{~T}$ & $\begin{array}{l}\% \\
\text { of } \\
\mathrm{V} \\
\text { ari } \\
\text { an } \\
\text { ce }\end{array}$ & $\begin{array}{l}\text { Cum } \\
\text { ulati } \\
\text { ve } \%\end{array}$ \\
\hline 1 & $\begin{array}{l}3 \\
. \\
3 \\
3 \\
4\end{array}$ & $\begin{array}{r}33 \\
.3 \\
38\end{array}$ & $\begin{array}{r}33.3 \\
38\end{array}$ & $\begin{array}{l}. \\
3 \\
3 \\
4\end{array}$ & $\begin{array}{r}33 \\
.3 \\
38\end{array}$ & $\begin{array}{r}33.3 \\
38\end{array}$ & 3 & $\begin{array}{r}32 \\
.7 \\
32\end{array}$ & $\begin{array}{r}32.7 \\
32\end{array}$ \\
\hline 2 & $\begin{array}{l}. \\
2 \\
7 \\
8\end{array}$ & $\begin{array}{r}22 \\
.7 \\
83\end{array}$ & $\begin{array}{r}56.1 \\
21\end{array}$ & 7 & $\begin{array}{r}22 \\
.7 \\
83\end{array}$ & $\begin{array}{r}56.1 \\
21\end{array}$ & 2 & $\begin{array}{r}21 \\
.8 \\
22\end{array}$ & $\begin{array}{r}54.5 \\
54\end{array}$ \\
\hline
\end{tabular}




\begin{tabular}{|c|c|c|c|c|c|c|c|c|c|}
\hline & \multicolumn{3}{|c|}{$\begin{array}{c}\text { Initial } \\
\text { Eigenvalues }\end{array}$} & \multicolumn{3}{|c|}{$\begin{array}{l}\text { Sums of } \\
\text { Squared } \\
\text { Loadings }\end{array}$} & \multicolumn{3}{|c|}{$\begin{array}{c}\text { Rotation Sums } \\
\text { of Squared } \\
\text { Loadings }\end{array}$} \\
\hline 3 & 6 & $\begin{array}{r}16 \\
.9 \\
17\end{array}$ & $\begin{array}{r}73.0 \\
38\end{array}$ & $\begin{array}{l}1 \\
. \\
6 \\
9 \\
2\end{array}$ & $\begin{array}{r}16 \\
.9 \\
17\end{array}$ & $\begin{array}{r}73.0 \\
38\end{array}$ & $\begin{array}{l}. \\
8 \\
4 \\
8\end{array}$ & $\begin{array}{r}18 \\
.4 \\
84\end{array}$ & $\begin{array}{r}73.0 \\
38\end{array}$ \\
\hline 4 & 1 & $\begin{array}{r}9 . \\
88 \\
0\end{array}$ & $\begin{array}{r}82.9 \\
18\end{array}$ & 1 & $\begin{array}{r}9 . \\
88 \\
0\end{array}$ & $\begin{array}{r}82.9 \\
18\end{array}$ & $\begin{array}{l}3 \\
2 \\
5\end{array}$ & $\begin{array}{r}9 . \\
88 \\
0\end{array}$ & $\begin{array}{r}82.9 \\
18\end{array}$ \\
\hline 5 & 2 & $\begin{array}{r}6 . \\
28 \\
3\end{array}$ & $\begin{array}{r}89.2 \\
01\end{array}$ & & & & & & \\
\hline 6 & $\begin{array}{l}5 \\
0 \\
1\end{array}$ & $\begin{array}{r}5 . \\
00 \\
5\end{array}$ & $\begin{array}{r}94.2 \\
06\end{array}$ & & & & & & \\
\hline 7 & $\begin{array}{l}. \\
4 \\
0 \\
3\end{array}$ & $\begin{array}{r}4 . \\
02 \\
6\end{array}$ & $\begin{array}{r}98.2 \\
32\end{array}$ & & & & & & \\
\hline 8 & $\begin{array}{l}1 \\
2 \\
2\end{array}$ & $\begin{array}{r}1 . \\
22 \\
1\end{array}$ & $\begin{array}{r}99.4 \\
53\end{array}$ & & & & & & \\
\hline 9 & $\begin{array}{l}0 \\
3 \\
7\end{array}$ & $\begin{array}{l}.3 \\
69\end{array}$ & $\begin{array}{r}99.8 \\
22\end{array}$ & & & & & & \\
\hline 10 & $\begin{array}{l}0 \\
1 \\
8\end{array}$ & $\begin{array}{l}.1 \\
78\end{array}$ & $\begin{array}{r}100 . \\
000\end{array}$ & & & & & & \\
\hline
\end{tabular}

We can see that, four eigenvalues of common factors were greater than 1.00, and the cumulative variance contribution rates of them for three consecutive years were $80.616 \%, 81.855 \%$ and $82.918 \%$ respectively, which indicated that these four common factors could describe the profitability of modern logistics enterprise comprehensively.

\section{Comprehensive Analysis of the Profitability of Modern Logistics Enterprise}

Component score coefficient matrix for three years were shown in Table 5. Four common factors are operational management capacity, profitability, debt repayment capacity and capacity of future development. We could see that there was a cross between different factors in different degrees, these factors reflected the comprehensive profitability of listed company, and using any common factor alone could not make a comprehensive analysis of the profitability of listed companies in modern logistics enterprise.

TABLE V. COMPONENT COEFFICIENT MATRIX FROM 2010 TO 2012

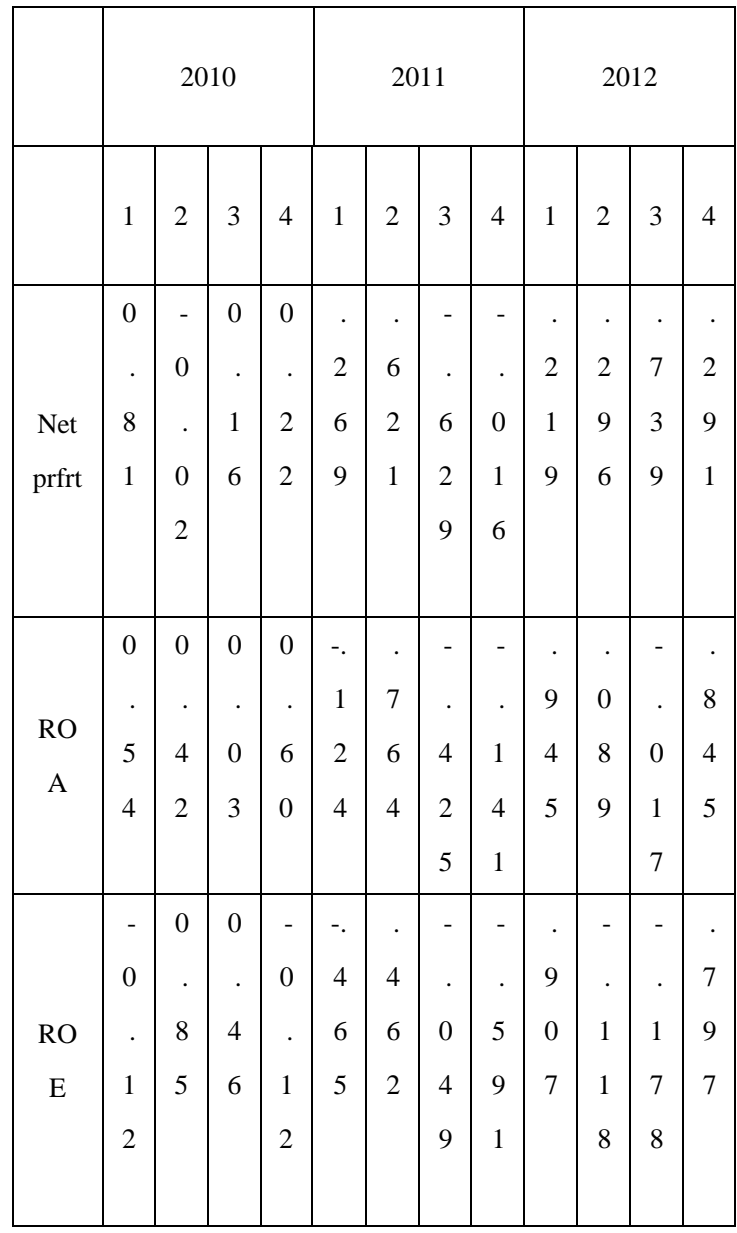




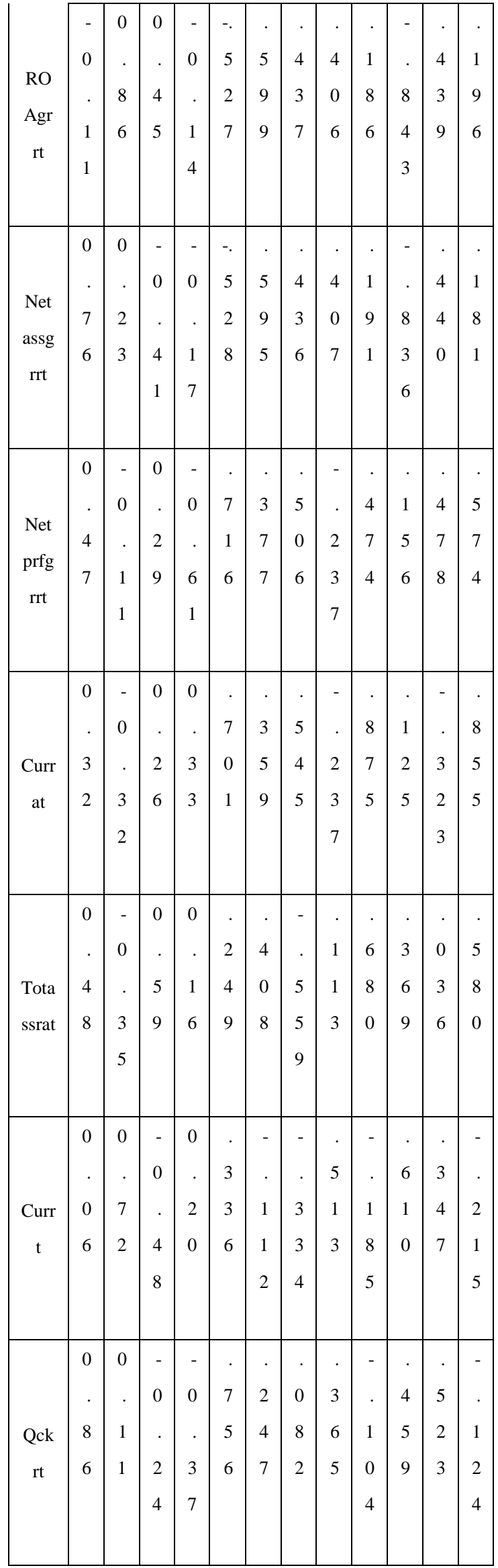

We use the variance contribution rates as a weight for the weighted average calculation to carry on the comprehensive analysis and evaluation of the profitability of modern logistics enterprise. The top 15 of 73 in the comprehensive factor scores for three consecutive years were showed in Table 6. Most of them were in transportation (road, rail, aviation, marine transportation), and were mainly located in coastal and economically developed areas, such as Guangdong, Hainan, Fujian, Shandong, Jiangsu, Shanghai, which indicated that the modern logistics enterprises in marine transportation had the strongest comprehensive profitability.

TABLE VI. THE COMPARISON OF MODERN LOGISTICS ENTERPRISE PROFITABILITY

\begin{tabular}{|c|c|c|c|c|c|c|}
\hline $\begin{array}{l}\mathrm{R} \\
\mathrm{a} \\
\mathrm{n}\end{array}$ & \multicolumn{2}{|l|}{2010} & \multicolumn{2}{|l|}{2011} & \multicolumn{2}{|l|}{2012} \\
\hline 1 & $\begin{array}{l}\text { Hainan Strait } \\
\text { Shipping }\end{array}$ & $\begin{array}{l}9 \\
6\end{array}$ & $\begin{array}{l}\text { Wuhu Port } \\
\text { Storage }\end{array}$ & $\begin{array}{l}5 \\
2 \\
2\end{array}$ & $\begin{array}{l}\text { Tianjin } \\
\text { Marine } \\
\text { Shipping }\end{array}$ & 2 \\
\hline 2 & $\begin{array}{l}\text { Shenzhen } \\
\text { Yantian Port }\end{array}$ & $\begin{array}{l}1 \\
. \\
1 \\
6\end{array}$ & $\begin{array}{l}\text { Hainan } \\
\text { trait } \\
\text { hipping }\end{array}$ & $\begin{array}{l}5 \\
0 \\
4\end{array}$ & $\begin{array}{l}\text { Shandon } \\
\text { g Airlines }\end{array}$ & 8 \\
\hline 3 & $\begin{array}{l}\text { Hubei Yichang } \\
\text { Transportation } \\
\text { Group }\end{array}$ & 0 & $\begin{array}{l}\text { Zhongchu } \\
\text { developme } \\
\text { nt }\end{array}$ & $\begin{array}{l}. \\
8 \\
3 \\
0\end{array}$ & $\begin{array}{l}\text { Bohai } \\
\text { Ferry }\end{array}$ & 0 \\
\hline 4 & $\begin{array}{l}\text { Shandong } \\
\text { Airlines }\end{array}$ & 8 & $\begin{array}{l}\text { Shandong } \\
\text { Airlines }\end{array}$ & $\begin{array}{l}. \\
4 \\
7 \\
4\end{array}$ & $\begin{array}{l}\text { Zhangjia } \\
\text { gang } \\
\text { Bonded } \\
\text { Technolo } \\
\text { gy }\end{array}$ & 7 \\
\hline
\end{tabular}




\begin{tabular}{|c|c|c|c|c|c|c|}
\hline & $\begin{array}{l}\text { Citroen } \\
\text { Railway } \\
\text { Container } \\
\text { Logistics }\end{array}$ & $\begin{array}{l}7 \\
8 \\
1\end{array}$ & $\begin{array}{l}\quad \text { Hubei } \\
\text { Yichang } \\
\text { Transportati } \\
\text { on Group }\end{array}$ & $\begin{array}{l}\cdot \\
4 \\
0 \\
3\end{array}$ & $\begin{array}{l}\text { Fujia } \\
\mathrm{n} \\
\text { Longzho } \\
\mathrm{u} \text { transpo } \\
\mathrm{rt}\end{array}$ & $\begin{array}{l}. \\
5 \\
5 \\
5\end{array}$ \\
\hline 6 & $\begin{array}{l}\text { Xiamen } \\
\text { International } \\
\text { Airport }\end{array}$ & $\begin{array}{l}0 \\
. \\
7 \\
2 \\
7\end{array}$ & $\begin{array}{l}\text { Fujian } \\
\text { Longzhou t } \\
\text { ransport }\end{array}$ & $\begin{array}{l}0 \\
. \\
3 \\
6 \\
9\end{array}$ & $\begin{array}{l}\text { Wuhu } \\
\text { Port } \\
\text { Storage }\end{array}$ & $\begin{array}{l}. \\
4 \\
7 \\
0\end{array}$ \\
\hline 7 & $\begin{array}{l}\text { China Eastern } \\
\text { Airlines }\end{array}$ & $\begin{array}{l}0 \\
. \\
6 \\
7 \\
2\end{array}$ & $\begin{array}{l}\text { Jiangsu } \\
\text { Aoyang Sh } \\
\text { unchang }\end{array}$ & $\begin{array}{l}0 \\
\cdot \\
3 \\
6 \\
7\end{array}$ & $\begin{array}{l}\text { Zhongch } \\
\text { u } \\
\text { developm } \\
\text { ent }\end{array}$ & $\begin{array}{l}. \\
4 \\
6 \\
0\end{array}$ \\
\hline 8 & $\begin{array}{l}\text { China Southern } \\
\text { Airlines }\end{array}$ & $\begin{array}{l}0 \\
. \\
6 \\
2 \\
2\end{array}$ & $\begin{array}{l}\text { Citroen } \\
\text { Railway } \\
\text { Container } \\
\text { Logistics }\end{array}$ & $\begin{array}{l}0 \\
. \\
3 \\
0 \\
2\end{array}$ & $\begin{array}{l}\text { Beihai } \\
\text { Port }\end{array}$ & $\begin{array}{l}. \\
4 \\
3 \\
0\end{array}$ \\
\hline 9 & $\begin{array}{l}\text { China } \\
\text { International } \\
\text { Aviation }\end{array}$ & $\begin{array}{l}0 \\
. \\
5 \\
6 \\
0\end{array}$ & Beihai Port & $\begin{array}{l}. \\
2 \\
7 \\
5\end{array}$ & $\begin{array}{l}\text { Daqin } \\
\text { Railway }\end{array}$ & $\begin{array}{l}\cdot \\
4 \\
2 \\
4\end{array}$ \\
\hline 0 & Bohai Ferry & $\begin{array}{l}0 \\
. \\
4 \\
8 \\
9\end{array}$ & $\begin{array}{l}\text { North } \\
\text { Freeway }\end{array}$ & $\begin{array}{l}\cdot \\
2 \\
4 \\
2\end{array}$ & $\begin{array}{l}\text { Jiangsu } \\
\text { Aoyang } \\
\text { Shunchan } \\
\text { g }\end{array}$ & $\begin{array}{l}. \\
3 \\
9 \\
0\end{array}$ \\
\hline 1 & $\begin{array}{l}\text { Zhuhai Hengji } \\
\text { Daxin } \\
\text { international } \\
\text { chemical storag } \\
\text { e }\end{array}$ & $\begin{array}{l}0 \\
. \\
4 \\
5 \\
7\end{array}$ & $\begin{array}{l}\text { China } \\
\text { Eastern } \\
\text { Airlines }\end{array}$ & $\begin{array}{l}. \\
1 \\
7 \\
6\end{array}$ & $\begin{array}{l}\text { Xiamen } \\
\text { Internatio } \\
\text { nal } \\
\text { Airport }\end{array}$ & $\begin{array}{l}. \\
3 \\
8 \\
6\end{array}$ \\
\hline 2 & $\begin{array}{l}\text { Jiangsu } \\
\text { Aoyang Shunc } \\
\text { hang }\end{array}$ & $\begin{array}{l}0 \\
. \\
4 \\
4 \\
4\end{array}$ & $\begin{array}{l}\text { Sinotrans } \\
\text { Air } \\
\text { Transportat } \\
\text { ion } \\
\text { Developme } \\
\text { nt }\end{array}$ & $\begin{array}{l}. \\
1 \\
7 \\
2\end{array}$ & $\begin{array}{l}\text { China } \\
\text { Eastern } \\
\text { Airlines }\end{array}$ & $\begin{array}{l}\cdot \\
3 \\
5 \\
1\end{array}$ \\
\hline
\end{tabular}

\begin{tabular}{|c|c|c|c|c|c|}
\hline 3 & $\begin{array}{l}\text { Modern } \\
\text { Investment }\end{array}$ & 4 & $\begin{array}{l}\text { Xiamen } \\
\text { Port } \\
\text { Developme } \\
\text { nt }\end{array}$ & $\begin{array}{l}1 \\
5 \\
8\end{array}$ & $\begin{array}{l}\text { Shanghai } \\
\text { Shentong } \\
\text { Metro }\end{array}$ \\
\hline 4 & $\begin{array}{l}\text { Zhangjiagang } \\
\text { Bonded } \\
\text { Technology }\end{array}$ & 4 & $\begin{array}{l}\text { Shanghai } \\
\text { Shentong } \\
\text { Metro }\end{array}$ & 1 & $\begin{array}{l}\text { Xiamen } \\
\text { Port } \\
\text { Develop } \\
\text { ment }\end{array}$ \\
\hline 5 & $\begin{array}{l}\text { Shenzhen } \\
\text { Chiwan Wharf }\end{array}$ & 2 & $\begin{array}{l}\text { Jiangxi } \\
\text { Changyun }\end{array}$ & . & $\begin{array}{l}\text { Jiangxi } \\
\text { Changyu } \\
\text { n }\end{array}$ \\
\hline
\end{tabular}

\section{CONCLUSIONS}

From the results of factor analysis for three consecutive years(from 2010 to 2012), we can conclude that the comprehensive factors affecting the profitability of modern logistics enterprise include four aspects (ie., operational management capacity, profitability, debt repayment capacity and capacity of future development).Most of modern logistics enterprises that have the stronger profitability were in transportation (road, rail, aviation, marine transportation), and the strongest is in marine transportation. Thay are mainly located in coastal and economically developed areas, such as Guangdong, Hainan, Fujian, Shandong, Jiangsu, Shanghai , which shows that regional factors is one of the main factors affecting the profitability of modern logistics enterprises.

\section{REFERENCES}

[1] Ohlson, J. Earnings, "Book values and dividends in equity valuation, Contemporary Accounting Research, "vol.11,1995, pp.661-687.

[2] L. Xiaorong, W. Xianliang, "Factor analysis in profitability of listed companies Analysis, "Finance Science, vol.7,2002, pp. 218-220.

[3] Z. Jixiu, "An Empirical Study of the profitability of listed companies in China industry characteristics," Management Science, vol.6, 2004,pp.39-45.

[4] Z. Qingchang, F. Zaiyu, 'Analysis China listing Corporation profitability model," Journal of Guizhou University of Finance and Economics, vol.1, 2006, pp.41-45.

[5] L. Yizhi, Z. Ping, "Factor Analysis and Evaluation of China's securities companies operating condition," Economic and Management Research, vol.2,2002, pp.38-39. 
[6] M. Shenghong, L. Mingwei, "To establish a comprehensive evaluation model of listing Corporation profitability," Communication of Finance and accounting, vol.1,2007, pp.41-43.

[7] W. Yuanfang, X. Di, "An Empirical Study of the profitability of listed companies in China industry differences ,"Accounting Research, vol.20,2008, pp.47-49.

[8] Z. Lu, "Comprehensive Evaluation of Regional Logistics System Development," Academic Essays, vol.22,pp.2009,100- 102.

[9] L. Shengchun, "Countermeasures of Regional Logistics Development in Anhui Province Based on Factor Analysis," Logistics Technology, vol.4, 2008,pp.76- 78.

[10] S.Peng. L.Xinxing. "Empirical Study on the Synergies between Regional Logistics Service and Manufacturing Development," Collected Essays on Finance and Economics, vol.5,2012, pp.97-102. 